\title{
Cutting Planes Based Relaxed Optimal Power Flow in Active Distribution Systems
}

\author{
Hongjun Gao, ${ }^{\mathrm{a}, \mathrm{,},}$, Junyong Liu ${ }^{\mathrm{a}}$, Lingfeng Wang ${ }^{\mathrm{b}}$, Youbo Liu ${ }^{\mathrm{a}}$
}

${ }^{\mathrm{a}}$ School of Electrical Engineering and Information, Sichuan University, 610065, Chengdu, China.

${ }^{b}$ Department of Electrical Engineering and Computer Science, University of WisconsinMilwaukee, 53211, Milwaukee, WI, USA.

Corresponding author: Hongjun Gao: School of Electrical Engineering and Information, Sichuan University, Chengdu 610065, China; E-mail:gaogavin1989@gmail.com; Tel: +8615202864988

\begin{abstract}
Optimal power flow (OPF) has played a significant role in the design, planning and operation of active distribution systems (ADSs). Due to its ability to achieve the optimality with higher computational efficiency, the second order cone programming (SOCP) based on branch flow model (BFM) has received an increasing attention in recent years. However, various sufficient conditions and assumptions are required to ensure the relaxation exactness in the existing literatures. In this study, we introduce the cutting planes to tackle this exactness challenge for general distribution networks especially the ADS with high renewable penetration. Firstly, a typical operation optimization model is presented as an example of OPF in ADS. A general branch flow model based relaxed optimal power flow (BFM-ROPF) model is then formulated as a SOCP problem after conic relaxation. According to these conditions with the objectives which are not monotonously increasing in power injections or branch currents, a total
\end{abstract}


power loss cut (TPLC) is introduced to ensure the conic relaxation exactness. Moreover, a leaf branch current cut (LBCC) is incorporated to prevent the inexactness of SOCR in some leaf branches. Afterwards, the proof of the cutting planes is given to guarantee the optimality and the relaxation exactness of the BFM-ROPF model even though power loss is not included in the objectives. Numerical results based on 33-bus and 69-bus systems are given to verify the effectiveness of the proposed method.

Keywords: Active distribution system (ADS), second-order cone programming (SOCP), branch flow model (BFM), cutting planes, relaxation exactness.

The symbols used are defined in the following:

\section{Indices}
$i, j, h$
Index of buses.
$i j$
Index of branches.
$k$
Index of iteration.

Sets

${ }_{B} / B^{\text {Load }}$

$B^{\mathrm{ESS}} B^{\mathrm{WTG}} / B^{\mathrm{SVC}} / B^{\mathrm{TR}}$

$\delta(j)$

E

$\mathrm{P}_{i} / \mathrm{Q}_{i}$

Z

$\mathrm{Z}_{k}$

\section{Parameters}

$r_{i j} / x_{i j}$

$c^{\mathrm{Loss}}, c_{t}^{\mathrm{TR}}, c^{\mathrm{WTC}}$
Set of system buses/ load buses.

Set of buses connected with ESS/ WTG/ SVC/transformer.

Set of buses whose parent is bus $j$.

Set of branches.

Set of active/reactive power injections

The feasible set to the original model.

The feasible set in the $k$-th iteration.

Resistance/reactance of branch $i j$.

Price to power loss, main grid power and wind power 
curtailed.

$C^{\mathrm{Loss}}, C^{\mathrm{TR}}, C^{\mathrm{WTG}}, C \quad$ Cost due to power loss, main grid power and wind power curtailed, and total cost.

$\underline{P}_{j}^{\mathrm{TR}} / \bar{P}_{j}^{\mathrm{TR}}$

$\underline{Q}_{j}^{\mathrm{TR}} / \bar{Q}_{j}^{\mathrm{TR}}$

$\underline{P}_{j}^{\mathrm{ESS}} \bar{P}_{j}^{\mathrm{ESS}}$

$\bar{I}_{i j}$

$\underline{V}_{j} / \bar{V}_{j}$

$\underline{Q}_{j}^{\mathrm{SVC}} / \bar{Q}_{j}^{\mathrm{SVC}}$

$P_{j}^{\mathrm{WTG}, \mathrm{RRE}}$

$P_{j}^{\mathrm{Load}} / Q_{j}^{\mathrm{Load}}$

$\bar{l}_{i j} / \bar{v}_{j} / \underline{v}_{j}$

Variables

$P_{j}^{\mathrm{TR}}, P_{j}^{\mathrm{ESS}}$

$\Delta P_{j}^{\mathrm{WTG}}$

$Q_{j}^{\mathrm{TR}}, Q_{j}^{\mathrm{SVC}}$

$P_{i j} / Q_{i j}$

$I_{i j} / V_{j}$

$l_{i j} / v_{j}$

$p / q$

${ }_{P} / Q$
Lower/upper bound of main-grid active power at bus $j$.

Lower/upper bound of main-grid reactive power at bus $j$.

Lower/upper bound of power limit of ESS connected to bus $j$.

Current capacity limit of branch $i j$.

Lower/upper bound of voltage magnitude at bus $j$.

Lower/upper bound of reactive power for SVC connected to bus $j$.

Forecast output of wind power connected to bus $j$.

Active/reactive load for bus $j$.

The bound of branch current and bus voltage.

Active power for transformer bus, ESS bus $j$.

Wind power curtailed at for bus $j$.

Reactive power for transformer bus, CB bus, SVC bus $j$.

Active/reactive power flow from bus $i$ to bus $j$.

Current magnitude of branch $i j$ and voltage magnitude of bus $j$.

Square of current magnitude of branch $i j$ and square of voltage magnitude of bus $j$.

Vector of the active/reactive power injections.

Vector of the active/reactive powers in the branches. 


$$
\begin{array}{ll}
l / v & \text { Vector of the branch currents and bus voltages. } \\
\dot{x} & \text { The optimal solution of original model. } \\
\boldsymbol{x}_{k} & \text { The solution in the } k \text {-th iteration. }
\end{array}
$$

\section{Introduction}

With the higher penetration of distributed energy resource (DER) into the distribution system, the current passive distribution networks are being transformed into active distribution systems (ADS). As a result, the design, planning and operation of the contemporary distribution system are becoming much more complicated than the traditional one as various active devices should be scheduled simultaneously. Generally, optimal power flow (OPF) is a fundamental approach required to support decision-making by performing optimization for several objectives under certain physical and operating constraints [1-2]. Also, the initiative for promoting ADS demands more effective OPF methods with higher computing efficiency and higher solution accuracy.

In [3], the AC optimal power flow (AC-OPF) is emphasized due to the phenomena related to voltage magnitudes and reactive power which are of great relevance to distribution systems. Besides, in the distribution network some reasonable simplifications such as the linearization of power flow constraints which are commonly used in transmission systems are not applicable any more. Therefore, in order to address the challenges that the non-linear power flow equation constraints bring to distribution engineers, a considerable number of studies have been carried out on this topic which could be generally divided into two categories: heuristic algorithms and analytical methods [4-5]. The heuristic algorithms have been paid much attention due to their ease 
of implementation, which mainly include genetic algorithm [6], Tabu search [7], particle swarm optimization method [8], etc. But these heuristic algorithms have a few major disadvantages (e.g. high computing cost), which largely reduce their adaptability and effectiveness in handling problems of ADS equipped with massive DERs. Therefore, more studies are focused on exploring the analytical methods in these decades [9], which have been developed primarily to solve the OPF in transmission networks. Traditional analytical methods for solving the highly non-linear problem include quadratic programming [10], Newton Raphson [11], interior point method [12], Lagrangian Relaxation [13] etc. Compared to those traditional analytical methods, convex programming becomes widely used because it is able to achieve the optimal solution in polynomial time by the means of the convexification of power flow equations [14-16]. The semidefinite programming (SDP) was first introduced in [17] to solve the OPF for mesh networks based on the bus injections model. Compared to SDP, the second-order cone programming (SOCP) has attracted more attention for radial distribution networks because of its much lower computational complexity [18-19]. A SOCP based load flow model is first proposed in [20], where the convex optimization problem is solved by interior point methods. Moreover, another type of the SOC relaxation (SOCR) approach in radial distribution systems is investigated in [21] and [22], which is based on the branch flow model (BFM). However, it is just the relaxation process that may result in inexact solutions in this branch flow model based relaxed OPF (BFM-ROPF) [23-24]. The optimization result is meaningless when the SOCR is not exact. Some sufficient conditions are given in [25] and [26] for radial networks (e.g., 
not both constraints on active and reactive power injections are binding at both ends of a line).

For the active distribution system with high penetration of DERs, the effectiveness of the SOCR is also verified in [27] using four IEEE benchmark systems and realistic systems. But only under a few specific strong conditions, the relaxation is exact (e.g. the objective function is convex and monotonically increasing in each active power injection and the initial OPF is feasible [28]). Moreover, in order to accommodate more renewable energies [29-30], some other objectives (e.g. renewable energy curtailment) should be taken into account in ADS operations, which may lead to the inexactness of SOCR because the objective function is not monotonically increasing in power injections of these buses with renewable energies. Meanwhile, it is common to include the penalty cost of renewable energy curtailed in objective functions to represent the interests of DG operators in a number of ADS studies.

As mentioned earlier, though many restrictive assumptions are given to guarantee the exactness of SOCR, no recourse is provided to obtain a feasible solution if these conditions are not satisfied or the computing results are not exact. A spatial branch and bound algorithm is proposed in [31] to achieve the optimality of SDP in several standard IEEE systems without any premises. However, it may be fairly difficult to compute the upper bound of the original nonlinear problem in the practical distribution networks by using CONOPT, which is a nonlinear programming (NLP) solver. Another trial implementation is an increasingly tight linear cut applied to the relaxed model. The cutting planes are previously applied to solve the relaxation problems for integer 
programming. In each iteration, a set of linear cuts is added until the convergence tolerance criterion is fulfilled. The cuts can eliminate the solution returned in the previous iteration from the feasible set when the relaxation is not exact. The cutting planes are first studied in [28] to ensure the exactness of relaxed optimal power flow in the radial distribution system. The relaxation gap may shrink to meet the desired requirement after several iterations. Yet, in [28] the study is conducted based on the premise that the relaxation is exact when the power loss is the single objective. Actually, in the practical test there may exist some SOCR gaps at some leaf branches even though the power loss is the single objective in the OPF model. In this work, we focus on obtaining the optimal solution in a given distribution system without any restrictive assumptions or sufficient conditions based on BFM-ROPF. First, a typical optimization model by BFM is built as an OPF example in ADS, where the high penetration of renewable energies is especially considered. The SOCP problem is then formulated for the general BFM-based OPF model after SOCR. In addition, in order to meet the exactness requirement, cutting planes including the total power loss cut (TPLC) and leaf branch current cut (LBCC) are applied to the relaxed OPF model in the iteration process. Then, the validity of cutting planes is proved to ensure the SOCR exactness for general situations. Afterwards, the applicability of the proposed cutting planes for relaxation exactness is investigated through carrying out numerical case studies. Specifically, the main contributions of this paper are threefold. First, a typical operation optimization model is used as an example in ADS with high penetration of renewable energies and active network management devices. Then the general relaxed 
optimal power flow model based on BFM is generated after the conic relaxation. Second, two types of cutting planes including TPLC and LBCC are introduced to ensure the exactness of the relaxed OPF model in the general conditions where power losses may not be included in the optimization objectives. Third, the influences of voltage constraints, main-grid constraints, and different penetration of WTG power on the optimization results are analyzed based on 33-bus and 69-bus systems. Moreover, the computational performances of cutting planes in different objectives are also thoroughly examined to validate the effectiveness of the proposed cutting planes.

The rest of the paper is organized as follows: In Section 2, a typical operation optimization model in ADS is presented. Section 3 generalizes the basic BFM-ROPF model. Section 4 gives two types of cutting planes and the proof of the validity of cutting planes for relaxation exactness in general radial distribution systems. Numerical computational results and analysis based on the 33-bus and 69-bus systems are presented in Section 5. Finally, conclusions are drawn in Section 6.

\section{A Typical Operation Optimization Model in ADS}

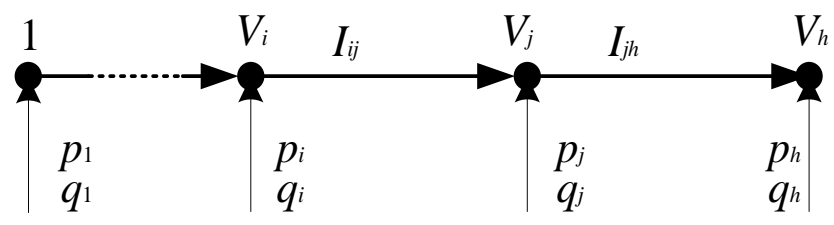

Fig. 1. Diagram for radial distribution network.

In this section, we describe a typical operation model in ADS with high penetration of renewable energies and active network management devices (e.g. static var compensator (SVC) and energy storage system (ESS)). The steady operation modes are almost radial (tree) in distribution systems, which is shown in Fig. 1. Generally, in the 
directional graph branch $i j$ implies that the positive direction of power flow is from bus $i$ to bus $j$. static var compensators (SVCs)

\subsection{Objective function}

The power loss is a common objective in the existing studies. For the distribution system operator, the total electricity purchasing cost should be minimized in the practical operation. Meanwhile, $C^{\mathrm{TR}}$ denotes electricity selling benefits if $C^{\mathrm{TR}}<0$. Furthermore, the punishment cost for wind power curtailed is included for promoting the consumption of renewable energies (wind power as an example in this study). So the total cost can be expressed as follows:

$$
\begin{aligned}
& C^{\mathrm{Loss}}=\sum_{i j \in E} c^{\mathrm{Loss}} I_{i j}^{2} r_{i j} \\
& C^{\mathrm{WTG}}=\sum_{j \in B^{\mathrm{WTG}}} c^{\mathrm{WTG}} \Delta P_{j}^{\mathrm{WTG}} \\
& C^{\mathrm{TR}}=\sum_{j \in B^{\mathrm{TR}}} c^{\mathrm{TR}} P_{j}^{\mathrm{TR}} \\
& \min C=C^{\mathrm{Loss}}+C^{\mathrm{WTG}}+C^{\mathrm{TR}}
\end{aligned}
$$

\subsection{Constraints}

$$
\begin{aligned}
& P_{i j}=-p_{j}+I_{i j}^{2} r_{i j}+\sum_{h \in \delta(j)} P_{j h}, \forall j \in B \\
& Q_{i j}=-q_{j}+I_{i j}^{2} x_{i j}+\sum_{h \in \delta(j)} Q_{j h}, \forall j \in B \\
& V_{i}^{2}-V_{j}^{2}=2\left(P_{i j} r_{i j}+Q_{i j} x_{i j}\right)-I_{i j}^{2}\left(r_{i j}^{2}+x_{i j}^{2}\right), \forall i j \in E \\
& I_{i j}^{2} V_{i}^{2}=P_{i j}^{2}+Q_{i j}^{2}, \forall i j \in E \\
& \underline{V}_{j} \leq V_{j} \leq \bar{V}_{j}, \forall j \in B \\
& 0 \leq I_{i j} \leq \bar{I}_{i j}, \forall i j \in E \\
& \underline{P}_{j}^{\mathrm{TR}} \leq P_{j}^{\mathrm{TR}} \leq \bar{P}_{j}^{\mathrm{TR}} \\
& \underline{Q}_{j}^{\mathrm{TR}} \leq Q_{j}^{\mathrm{TR}} \leq \bar{Q}_{j}^{\mathrm{TR}}, \forall j \in B^{\mathrm{TR}} \\
& \underline{Q}_{j}^{\mathrm{SVC}} \leq Q_{j}^{\mathrm{SVC}} \leq \bar{Q}_{j}^{\mathrm{SVC}}, \forall j \in B^{\mathrm{SVC}} \\
& \underline{P}_{j}^{\mathrm{ESS}} \leq P_{j}^{\mathrm{ESS}} \leq \bar{P}_{j}^{\mathrm{ESS}}, \forall j \in B^{\mathrm{ESS}}
\end{aligned}
$$




$$
\begin{aligned}
& 0 \leq \Delta P_{j, t}^{\mathrm{WTG}} \leq P_{j, t}^{\mathrm{WTG}, \mathrm{PRE}}, \forall t, \forall j \in B^{\mathrm{WTG}} \\
& p_{j}=P_{j}^{\mathrm{WTG}, \mathrm{PRE}}-\Delta P_{j}^{\mathrm{WTG}}+P_{j}^{\mathrm{TR}}+P_{j}^{\mathrm{ESS}}-P_{j}^{\mathrm{Load}}, \forall j \in B \\
& q_{j}=Q_{j}^{\mathrm{TR}}+Q_{j}^{\mathrm{SVC}}-Q_{j}^{\mathrm{Load}}, \forall j \in B
\end{aligned}
$$

The equations (2a-2d) represent the power flow constraints using distflow based branch flow model, which is first proposed in [32] and [33]. Equation (3) is the security constraints to assure the magnitude of the bus voltage and branch current which are kept within some permitted limits. Equation (4) keeps active and reactive power from main-grid in some limits for stability reasons of the transmission network. The future ADS may allow the distribution system operators to sell the surplus electricity to upper grid to gain some profits then $P_{j}^{\mathrm{TR}}<0$. Equation (5) and (6) represent the constraints on SVC and ESS, respectively. They are included as active elements in ADS for promoting the consumption of renewable energies. Equation (7) is the constraint of WTG power curtailed, from which we can find that this model allows for some renewable energy curtailment. Equation (8) and (9) represent the active power and reactive power injections in each bus. They are actually the supplements of equations (2a) and (2b).

\section{BFM Based Relaxed Power Flow Model in ADS}

According to [1], the optimization model (1)-(9) essentially describes an AC optimal power flow (AC-OPF) problem which is difficult to solve because of the nonlinear characteristics of power flow equations (2a)-(2d). Using $\boldsymbol{l}$ and $\boldsymbol{v}$, the power flow equations can be converted to the following form [18-19]:

$$
\begin{aligned}
& P_{i j}=-p_{j}+l_{i j} r_{i j}+\sum_{h \in \delta(j)} P_{j h}, \forall j \in B \\
& Q_{i j}=-q_{j}+l_{i j} x_{i j}+\sum_{h \in \delta(j)} Q_{j h}, \forall j \in B
\end{aligned}
$$




$$
\begin{aligned}
& v_{i}-v_{j}=2\left(P_{i j} r_{i j}+Q_{i j} x_{i j}\right)-l_{i j}\left(r_{i j}^{2}+x_{i j}^{2}\right), \forall i j \in E \\
& l_{i j} v_{i}=P_{i j}^{2}+Q_{i j}^{2}, \forall i j \in E
\end{aligned}
$$

However, after the above transformation the power flow model is still nonconvex due to quadratic equalities in (10d). Then the conic relaxation is conducted to convert (10d) to inequalities whose second order cone form can be expressed as

$$
\left\|\begin{array}{l}
2 P_{i j} \\
2 Q_{i j} \\
l_{i j}-v_{i}
\end{array}\right\|_{2} \leq l_{i j}+v_{i}, \forall i j \in E
$$

After the conic relaxation, the general BFM-ROPF can be expressed as follows:

$$
\begin{aligned}
& \min \sum_{i \in B} f_{i}\left(p_{i}\right) \\
& \text { over } \boldsymbol{p}, \boldsymbol{q}, \boldsymbol{P}, \boldsymbol{Q}, \boldsymbol{v}, \boldsymbol{l} \\
& \text { s.t. }(10 \mathrm{a})-(10 \mathrm{c}),(11) \\
& p_{i} \in \mathrm{P}_{i}, q_{i} \in \mathrm{Q}_{i} \forall i \in B \\
& l_{i j} \leq \bar{l}_{i j}, \forall i j \in E \\
& \underline{v}_{i} \leq v_{i} \leq \bar{v}_{i}, \forall i \in B
\end{aligned}
$$

The model (12) is clearly an SOCP problem. (12b) shows the vectors of decision variables. For the objective functions, basically any one can be expressed as $\sum_{i \in B} f_{i}\left(p_{i}\right)$, especially for the power loss in the network when $f_{i}\left(p_{i}\right)=p_{i}$ for $\forall i \in B$. However, this study excludes some other objectives such as conservation of voltage reduction (CVR) which are researched in several studies [19]. As a matter of fact, the active and reactive power injections are the control variables $(\boldsymbol{p}, \boldsymbol{q})$ to which we usually pay more attention. Besides, these derivative variables (e.g. $P_{j}^{\mathrm{TR}}$ ) are essentially included in the control variables $(p, q)$. Therefore, the constraints related to control variables can be summarized in (12d). After specifying the control variables, the other variables which 
are called state variables can subsequently be determined.

Several sufficient conditions are shown in [26] to ensure the exactness of SOCR. And [27] presents a mild condition for the relaxed radial optimal power flow and generalizes the previous work. It is shown that these conditions may not hold in many practical networks and SOCR is accordingly not exact. From the viewpoint of geometry, [24] reveals that under some conditions such as not imposing both lower bound constraints on active power injections at both ends of a line, the relaxed model and the original model share the same Pareto front. More importantly, in the above literatures it is assumed that the objectives are strictly increasing in power injections or branch current. Obviously, $f_{i}\left(p_{i}\right)$ may not be a monotonously increasing function in active power injections in bus $i$, e.g. $C^{\mathrm{WTG}}$ in (1). Consequently, the optimization result is meaningless if the SOCR is not exact. Here, a brief illustration of the SOCR process is given in Fig. 2, where $S_{\text {original }}$ is the feasible set of the original model which is non-liner due to equation (10d) before relaxation. After the SOCR, the feasible region is enlarged to a convex set $S_{\text {SocR }}$ which obviously contains $S_{\text {original }}$. If the optimal solution returned by SOCP exactly lies in $S_{\text {original }}$, the relaxation is exact and the equation (11) is equivalent to (10d).

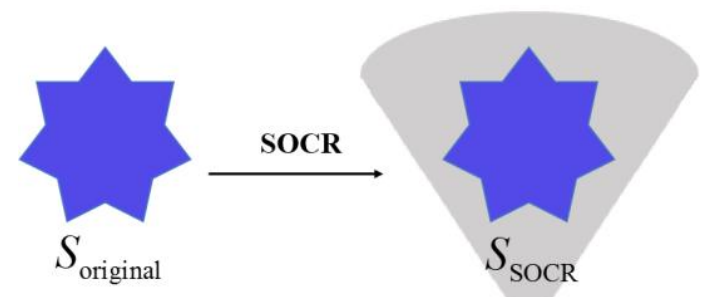

Fig. 2. The illustration of SOCR.

\section{Cutting Planes to Ensure the Exactness of SOCR}


From the previous work reported in [24] and [28], we find that two factors will have significant influences on the relaxation exactness, which are objective functions (see (12a)) and the feasible set of power injections (see (12d)) when almost the same constraints on the branch currents (see (12e)) and bus voltages (see (12f)) exist in almost every OPF study. The cutting planes can eliminate the inexact solution from the feasible set [28]. However, the study in [28] is based on the premise that the relaxation is definitively exact when the power loss is the single objective. Therefore, in this paper another cut (LBCC) is added to guarantee the relaxation exactness even though the power loss is the only objective. Then we focus on the general conditions where power loss may not be included in the optimization objectives. In this section, we first present two types of cuts (LBCC and TPLC). LBCC is included to prevent the inexactness of SOCR in some leaf branches. TPLC is added to promote the decreases of relaxation gap in each iteration until the computational requirement is fulfilled. Then we prove the proposed cutting planes are feasible in general conditions where the power loss may not be included in the optimization objectives. Finally, the detailed procedure of the relaxed OPF with cutting planes is presented.

\subsection{Cutting Planes}

Previously, the cutting planes are mainly applied to solve the relaxation problems for integer programming. They eliminate the solution returned in the previous iteration from the feasible set via adding a set of linear cuts when the relaxation is not exact or equivalent to the original non-relaxed model. Meanwhile, the optimal solution of the original non-relaxed problem must always be kept in the feasible set. At each iteration, 
new cuts are added until the convergence tolerance criterion is fulfilled.

\subsubsection{LBCC}

Here, the relative gap of the SOC relaxation at branch $i j$ in $k$-th iteration can firstly be defined as

$$
\Delta_{i j, k}^{\mathrm{diff}}=\frac{l_{i j, k} v_{i, k}-\left(P_{i j, k}^{2}+Q_{i j, k}^{2}\right)}{l_{i j, k} v_{i, k}}
$$

The maximum relative gap of the SOC relaxation in the $k$-th iteration can subsequently be defined as the maximum of the gaps at all branches, which is

$$
\Delta_{k}^{\mathrm{diff}}=\max \left\{\Delta_{i j, k}^{\mathrm{diff}}, \forall i j \in E\right\}
$$

From [34], we know that provided the feasible set of power injections is non-empty and the power loss is the only optimization objective, the SOC relaxation is exact for radial (tree) networks. In practical testing, we find out that there exist small gaps of relaxation in some leaf branches even though the power loss is the single objective in the programming model. The simple cut, however, can be integrated into the model to ensure the relative exactness of SOCR. The cut in the $k$-th iteration is as follows:

$$
l_{i j} \leq \frac{P_{i j, k-1}^{2}+Q_{i j, k-1}^{2}}{v_{i, k-1}}, \forall i j \in E_{k-1}^{L}
$$

where $E_{k-1}^{L}$ is the set of leaf lines whose relaxation results are not exact in the iteration $k$-1. This cut is named as LBCC as it mainly restricts the currents at leaf branches in the $k$-th iteration.

\subsubsection{TPLC}

From the previous analysis, we can summarize that the relaxation is always exact for radial networks if only power loss is included in the objective function when cutting 
plane (15) is introduced. In [17], it is assumed that the objective function is strictly increasing in each active power injections. It is not realistic to just minimize the power loss, some other objectives may be considered in many studies related to active distribution systems. However, in ADS, these objectives (e.g. renewable energy curtailment) may not be monotonously increasing in power injections (e.g. $C^{\text {WTG }}$ in (1)). Obviously, in the above situations, the SOCR is not exact.

For brevity, to describe the theory of cutting planes, we define

$$
\begin{aligned}
& I(\boldsymbol{x})=\sum_{i j \in E} r_{i j} l_{i j} \\
& L(\boldsymbol{x})=\sum_{i j \in E} r_{i j} \frac{P_{i j}^{2}+Q_{i j}^{2}}{v_{i}}
\end{aligned}
$$

Consequently, the cutting plane which promotes an increasing tight exactness of SOC relaxation can be expressed as

$$
I(\boldsymbol{x}) \leq L\left(\boldsymbol{x}_{k-1}\right)
$$

We call (18) TPLC and add it to the relaxed model in the $k$-th iteration. Therefore, the cut (15) and (18) constitutes the cutting planes as a whole in this study.

\subsection{Proof of Cutting Planes for General Conditions}

In [28], the validity of the cuts has been verified when the power loss is one of the optimization objectives. Here, via a simple modification we would like to extend the application of the cutting planes to OPF optimization in ADS where power loss may even be excluded in objective functions when the interest of DG operators or maximization utilization of renewable energies is the only target. Luckily, almost all the objective functions can be expressed as (12a).

Here, we can rewrite the objective function (12a) as 


$$
\sum_{i \in B} f_{i}\left(p_{i}\right)=\sum_{i \in B} p_{i}+\sum_{i \in B}\left(f_{i}\left(p_{i}\right)-p_{i}\right)
$$

where $\sum_{i \in B} p_{i}$ is the power loss in the system. It is equivalent to the traditional form which is shown as

$$
\begin{aligned}
& \sum_{i \in B} p_{i}=\sum_{(i, j) \in E} r_{i j} l_{i j} \\
& \sum_{i \in B} f_{i}\left(p_{i}\right)=\sum_{(i, j) \in E} r_{i j} l_{i j}+\sum_{i \in B}\left(f_{i}\left(p_{i}\right)-p_{i}\right)
\end{aligned}
$$

From (21), we find that nearly all objectives can be rewritten as a whole of two parts, one of which is actually the power loss. Therefore, the cutting planes are intended to be applied to the general conditions where the power loss may even be excluded from the optimization objectives.

$\mathrm{Z}_{k}$ is the set of feasible solutions of SOC relaxation minus the points eliminated by the cutting planes added in the step $k$. The target of cutting planes is to eliminate the current optimal solution from the previous relaxation set $Z_{k-1}$ which is convex and feasible due to the facts which are:

1. The set of feasible solutions to the original model $\mathrm{Z}$ is not empty.

2. The solution obtained in the iteration $k-1$ is not exact.

Because the second fact indicates $L\left(\boldsymbol{x}_{k-1}\right)$ is strictly inferior to $I\left(\boldsymbol{x}_{k-1}\right)$, the cuts (18) added to the $k$-th iteration can guarantee that the current optimal result will be eliminated from the feasible set. In the following we only need to prove that the optimal solution $\dot{\boldsymbol{x}}$ of the original OPF model is still kept in $\mathrm{Z}_{k}$ after cutting planes integration in any step $k$. Namely, the following condition must hold in each step:

$$
I(\dot{\boldsymbol{x}}) \leq L\left(\boldsymbol{x}_{k-1}\right), \quad \forall k
$$

Here, we know that the original case for $k=0$ is not exact and it is obvious that 
$\mathrm{Z} \subset \mathrm{Z}_{0}$.

According to [28], the following relationship must hold at first.

$$
I(\dot{\boldsymbol{x}}) \leq I\left(\boldsymbol{x}_{k-1}\right), \quad \forall k
$$

Because $\dot{\boldsymbol{x}}$ belongs to $\mathrm{Z}_{k-1}$, the objective of $\boldsymbol{x}_{k-1}$ must not be greater than that $\dot{\boldsymbol{x}}$, that is

$$
\sum_{(i, j) \in E} l_{i j, k-1} r_{i j}+\sum_{i \in B}\left(f_{i}\left(p_{i, k-1}\right)-p_{i, k-1}\right) \leq \sum_{(i, j) \in E} \dot{i}_{i j} r_{i j}+\sum_{i \in B}\left(f_{i}\left(\dot{p}_{i}\right)-\dot{p}_{i}\right)
$$

For brevity, we define

$$
\Delta(\boldsymbol{x})=\sum_{i \in B}\left(f_{i}\left(p_{i}\right)-p_{i}\right)
$$

Combining (24) with (23), we can obtain

$$
\Delta(\dot{\boldsymbol{x}}) \geq \Delta\left(\boldsymbol{x}_{k-1}\right), \quad \forall k
$$

Any objective function in ADS can be expressed as a linear combination of $\Delta(\boldsymbol{x}), L(\boldsymbol{x})$ and $\operatorname{Gap}(\boldsymbol{x})$, that is

$$
\begin{aligned}
& \sum_{i \in B} f_{i}\left(p_{i}\right)=I(\boldsymbol{x})+\Delta(\boldsymbol{x})=G a p(\boldsymbol{x})+L(\boldsymbol{x})+\Delta(\boldsymbol{x}) \\
& G a p(\boldsymbol{x})=I(\boldsymbol{x})-L(\boldsymbol{x})
\end{aligned}
$$

As for (18), it can be clearly seen that $\boldsymbol{x}_{k-1}$ must be a Pareto efficient solution for the multi-objective function $[\Delta(\boldsymbol{x}), L(\boldsymbol{x}), \operatorname{Gap}(\boldsymbol{x})]$. Then

$$
\boldsymbol{x}_{k-1}=\underset{\substack{x \in Z_{k-1} \\ L(\boldsymbol{x}) \leq L\left(\boldsymbol{x}_{k-1}\right) \\ \Delta(\boldsymbol{x}) \leq \Delta\left(\boldsymbol{x}_{k-1}\right)}}{\operatorname{argmin}} \operatorname{Gap}(\boldsymbol{x})
$$

As we know $\operatorname{Gap}(\boldsymbol{x}) \geq 0$ and $\operatorname{Gap}(\dot{\boldsymbol{x}})=0, \dot{\boldsymbol{x}}$ is also a Pareto efficient solution of the above multi-objective programming problem. Then

$$
\dot{\boldsymbol{x}}=\underset{\substack{x \in Z_{k-1} \\ L(\boldsymbol{x} \leq L(\boldsymbol{x})}}{\operatorname{argmin}} \operatorname{Gap}(\boldsymbol{x})
$$

From the second fact mentioned previously, the following relationship obviously 
holds:

$$
\operatorname{Gap}(\dot{\boldsymbol{x}})<\operatorname{Gap}\left(\boldsymbol{x}_{k-1}\right)
$$

Combining (31) with (26), we can obtain:

$$
L(\dot{\boldsymbol{x}})<L\left(\boldsymbol{x}_{k-1}\right)
$$

Actually, the fact that $\operatorname{Gap}(\dot{\boldsymbol{x}})$ is zero leads to the following equation:

$$
I(\dot{\boldsymbol{x}})=L(\dot{\boldsymbol{x}})<L\left(\boldsymbol{x}_{k-1}\right)
$$

To this point, we complete the proof of the cutting planes from which it can be found that the cutting planes are valid, provided that the objective is convex and the feasible set of the original model is not empty. We extend the method in [28] to general optimization cases in ADS even if the power loss is not included in the objectives because nearly the whole objectives can actually be rewritten as (12a). Moreover, we add the new cut (15) to guarantee the relaxation absolutely exact. However, what we need to be aware of is that some special objectives (e.g. CVR) are excluded in this study because they cannot be transformed to the form in (12a). These situations are only involved in a few studies.

\subsection{Overall Procedure of the Relaxed OPF with Cutting Planes Integration}

The overall procedure to solve the BFM-ROPF in general radial networks is summarized as follows.

Step 1: Solve the BFM-ROPF (12) to get the solution $\boldsymbol{x}_{0}$.

Step 2: If $\boldsymbol{x}_{0}$ satisfies (10d) which requires the SOC relaxation exactness, stop and return $\boldsymbol{x}_{0 .}$. Otherwise, integrate the cuts to facilitate the exactness of SOC relaxation.

Step 3: If the power loss is the unique objective, add LBCC (15) to the relaxed OPF 
model. Otherwise, go to computing process considering LBCC (15) and TPLC (18), and set $k=1$.

Step 4: Solve the master BFM-ROPF model with (15) and (18).

Step 5: If $\Delta_{k}^{\text {diff }}<\varepsilon$, stop and return $\boldsymbol{x}_{k}$. Otherwise, update $k=k+1$ and go to Step 4 .

\section{Case Studies}

Two radial distribution networks are used to test the proposed method including IEEE 33-bus and PG\&E 69-bus. All the algorithms are executed on a $1.6 \mathrm{GHz}$ personal computer with 4 GB RAM, and the proposed method is programmed in Matlab R2014a where the SOCP is solved by CPLEX 12.6.0.

In this section, we mainly use these two test networks to analyze the influence of voltage constraints and main-grid constraints on optimization results. Moreover, the influence of different penetration of WTG power on relaxation exactness and operation cost is studied. It is noticeable that in the sections 5.2-5.4 the objective function is the total cost $C$ as shown in (1). Finally, the comparison of the computation performance using different objective functions are made to validate the effectiveness of cutting planes.

\subsection{Configurations of Two Test systems and Base Data}

The network parameters of these two test systems can be obtained from the existing literatures (e.g. [4]). Both systems have the same parameters for SVC and ESS which, however, are of different quantities in each system. There are two types of SVCs whose capacities are [-0.2 0.6] and [-0.1 0.3] Mvar, respectively. Moreover, for brevity, the charge and discharge power limit of ESSs are assumed to be the same in this paper. 
There are also two types of ESSs whose power limits are 0.3 and $0.1 \mathrm{MW}$, respectively. And the detailed parameters for SVC, ESS, and WTG access to each system are given in Table 1, where the numbers outside the parentheses are the bus nodes and those inside the parentheses are the types or quantities of equipment connected. And the cost of power loss is $200 \$ / \mathrm{MWh}$. The penalty cost of WTG power curtailed is $500 \$ / \mathrm{MWh}$. The substation bus voltage is fixed at 1.0 p.u. The lower and upper bounds of the main-grid active power are 0.5 and $5 \mathrm{MW}$, respectively. The forecast power of WTGs in each bus for the base case is $0.8 \mathrm{MW}$. The voltage bound for the base case in each bus is $[0.94,1.06]$. And the current limit for the base case in each branch is 400A. The transmission power cost is $47.47 \$ / \mathrm{MWh}$. The convergence tolerance $\varepsilon$ is 0.01 .

Table 1. Data for some new elements

\begin{tabular}{ccc}
\hline \hline Types & IEEE 33-bus system & PG 69-bus system \\
\hline SVC & $5(2), 15(2), 31(1)$ & $3(1), 11(2), 19(2), 36(1)$, \\
ESS & $16(1), 33(2)$ & $41(2), 53(2), 64(1)$ \\
WTG & $4,18,22$ & $2(1), 12(2)$ \\
\hline \hline
\end{tabular}

\subsection{Analysis on Influence of Voltage Constraints on Optimization Results}

Table 2. Comparison of results in different voltage bounds

\begin{tabular}{ccc|cc}
\hline \hline \multirow{2}{*}{$\begin{array}{c}\text { Upper } \\
\text { bound }\end{array}$} & \multicolumn{2}{c|}{ 33-bus } & \multicolumn{2}{c}{ 69-bus } \\
\cline { 2 - 5 } & Iter. & $C(\$)$ & Iter. & $C(\$)$ \\
\hline 1.03 & - & - & - & - \\
1.04 & 1 & 59.85 & 11 & 150.57 \\
1.05 & 1 & 59.85 & 12 & 150.76 \\
1.06 & 1 & 59.85 & 12 & 150.73 \\
1.07 & 1 & 59.85 & 11 & 150.43 \\
\hline \hline
\end{tabular}

The results concerning the number of iterations and total cost for both 33-bus and 69-bus system in different voltage bounds are listed in Table 2 where we can find out that for simpler system no cutting planes are required while for 69-bus system cutting planes are necessary for the relaxation exactness because of the relatively high 
penetration of wind power in the 69-bus system compared to that in the 33-bus system. The total cost hardly changes with the voltage bound. There, however, exists a voltage bound under which the optimization model is infeasible for both systems.

Furthermore, as we know that PV nodes (fixed voltage nodes) are difficult to handle in power flow calculation for distribution systems, this study will verify whether BFM-ROFP is suitable for multiple PV nodes when some buses with special DGs connected are needed to maintain at a fixed voltage level using the 33-bus system where we set the voltage in WTG bus 22 fixed at 1.0 p.u.

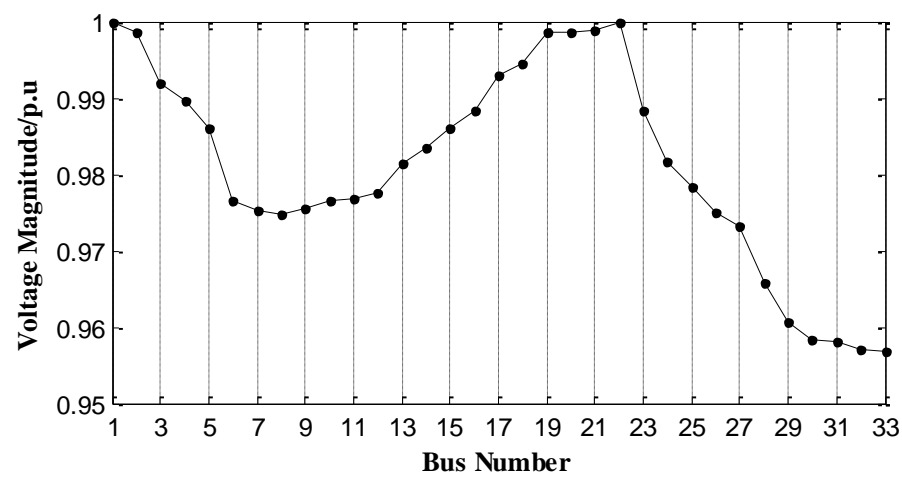

Fig. 3. Voltage distribution in each bus for the 33-bus system

Table 3. Optimization results in each iteration when voltage of bus 22 is fixed for the 33-bus system.

\begin{tabular}{c|c|c}
\hline \hline Iteration & $\Delta_{k}^{\text {diff }}$ & $C(\$)$ \\
\hline 1 & 87.4268 & 141.66 \\
2 & 8.3792 & 285.83 \\
3 & 0.0468 & 287.52 \\
4 & 0.0003 & 287.53 \\
\hline \hline
\end{tabular}

The detailed voltage value in each bus for the 33-bus system is shown in Fig. 3 where the voltage of bus 22 is 1.0 p.u. Furthermore, we can see that none of the voltages is greater than 1.0 p.u because the total wind power is $2.4 \mathrm{MW}$ which is less than the load demand. As depicted, the relative maximum gap is large with 87.4268 when cutting planes are not included. After cutting planes are introduced, the gap decreases quickly 
to 0.0003 in several iterations. So more iterations are required when some bus voltages are fixed, compared to the results in Table 2.

\subsection{Analysis on Influence of Main-grid Constraints on Optimization Results}

The main-grid constraints are crucial in the operation of both traditional distribution system (TDS) and ADS as for some security reasons. However, in the future ADS the reverse power flow is allowable and the distribution system operators can sell the surplus power to transmission network. In this section we set $\underline{P}_{j}^{\mathrm{TR}}=-5$ for the ADS case. The computational results for the 33-bus system in TDS are the same as those in ADS because the amount of wind power is not large enough to manifest the impact of main-grid constraints. The comparisons of optimization results including objective costs between TDS and ADS for the 69-bus system are shown in Table 4. We can observe that there exists some amount of wind power curtailed because of the technical constraints in TDS. On the contrary, the ADS can promote the consumption of renewable energies. Moreover, the model in ADS needs much fewer iterations because of looser restrictions and larger feasible regions.

Table 4. Comparison of results in TDS and ADS

\begin{tabular}{c|c|c|c|c}
\hline \hline Types & $C^{\text {Loss }}$ & $C^{\mathrm{WTG}}$ & $C^{\mathrm{TR}}$ & Iter. \\
\hline TDS & 14.61 & 112.38 & 23.74 & 12 \\
ADS & 15.24 & 0 & -24.76 & 2 \\
\hline \hline
\end{tabular}

\subsection{Optimization Results in Different Penetrations of WTGs}

The optimization results for 33-bus system in different penetration levels of wind power which is assumed to be the same in each bus are presented in the Table 5.

Table 5. Optimization results in different penetrations of wind power for the 33-bus system 


\begin{tabular}{c|c|c|c|c}
\hline \hline$P_{j}^{\mathrm{WTG}, \mathrm{PRE}}$ & $C^{\mathrm{Loss}}$ & $C^{\mathrm{WTG}}$ & $C^{\mathrm{TR}}$ & Iter. \\
\hline 0.6 & 12.35 & 0 & 74.85 & 1 \\
0.8 & 13.26 & 0 & 46.58 & 1 \\
1.0 & 14.87 & 0 & 23.74 & 1 \\
1.2 & 16.70 & 0 & 23.74 & 1 \\
1.4 & 18.35 & 246.63 & 23.74 & 9 \\
1.6 & 17.11 & 549.73 & 23.74 & 9 \\
\hline \hline
\end{tabular}

The optimization model proposed in this study allows certain wind power curtailment for economic and technical goals so that the model can still achieve feasible solutions as the forecast wind power in each bus reaches 1.6 MW. As the penetration of wind power in the power system increases, the total electricity purchasing cost from the main-grid will decrease accordingly. However, because of the main-grid constraints and the 3.7MW load demand in the 33-bus system the surplus wind power cannot be consumed when the wind power reaches 1.4 MW. Meanwhile, the reverse power flow will appear in case of a large percentage of renewable energies. From the iteration numbers shown in Table 5, we can observe that no more iterations are required when there is no wind power curtailed in the power system. In contrast, the cutting planes are necessary for relaxation exactness if much wind power is generated in the power system, which are also indicated for the 69-bus system shown in Table 6.

Table 6. Optimization results of ESS and SVC in different penetration levels of wind power for the 69-bus system

\begin{tabular}{c|c|c|c}
\hline \hline$P_{j}^{\text {WTG,PRE }}$ & ESS(MW) & SVC (Mvar) & Iter. \\
\hline 0.4 & -0.4 & 2.47 & 2 \\
0.6 & -0.38 & 2.47 & 2 \\
0.8 & 0.4 & 2.24 & 12 \\
1.0 & 0.4 & 2.28 & 13 \\
1.2 & 0.4 & 2.39 & 13 \\
\hline \hline
\end{tabular}

For the 69-bus system the optimization results of ESS and SVC in different 
penetration of wind powers are presented in Table 6 where we can observe that ESSs can discharge power to support the peak load demand while they could absorb some abundant power when the generation power exceeds the load demand in the local network. Consequently, ESSs play a significant role in adjusting the balance between power supply and demand as well as shifting loads to the valley periods. But as the WTGs cannot generate reactive power, there hardly appear surplus reactive power which may lead to risk of overvoltage - the total reactive power for SVCs remains stable in each situation.

Table 7. Optimization results in different penetration levels of wind powers for the 69-bus system

\begin{tabular}{c|c|c|c|c}
\hline \hline$P_{j}^{\mathrm{WTG}, \mathrm{PRE}}$ & $C^{\mathrm{Loss}}(\$)$ & $C^{\mathrm{WTG}}(\$)$ & $C^{\mathrm{TR}}(\$)$ & Iter. \\
\hline 0.4 & 17.84 & 0 & 70.80 & 2 \\
0.6 & 14.88 & 0 & 22.62 & 2 \\
0.8 & 15.24 & 0 & -24.76 & 2 \\
1.0 & 18.73 & 0 & -71.40 & 2 \\
1.2 & 25.15 & 0 & -117.35 & 2 \\
1.4 & 34.34 & 0 & -162.64 & 2 \\
\hline \hline
\end{tabular}

In addition, when we set $\underline{P}_{j}^{\mathrm{TR}}=-5$ in the main-grid constraints, the results for 69-bus system are shown in Table 7 where it can be seen that the BFM-ROPF model is also feasible even when the penetration of renewable energies is high enough to make operators to sell the electricity to the main-grid. And the model can converge in only 2 iterations. This facilitates the application of BFM-ROPF in the future ADS.

\subsection{Performance of Cutting Planes in Different Objectives}

In the previous parts, we analyze the influence of voltage and main-grid constraints on optimization results and find out that when high penetration of wind power and main-grid constraints appear in the system, the relaxation may not be exact if cutting 
planes are not integrated. For comparison purposes, seven cases with different objectives are listed in Table 8.

Table 8.Seven cases with different objectives

\begin{tabular}{|c|c|c|c|}
\hline Case & $C^{\text {Loss }}$ & $C^{\text {WTG }}$ & $C^{\mathrm{TR}}$ \\
\hline 1 & $\sqrt{ }$ & $\times$ & $\times$ \\
\hline 2 & $\times$ & $\sqrt{ }$ & $\times$ \\
\hline 3 & $\times$ & $\times$ & $\sqrt{ }$ \\
\hline 4 & $\sqrt{ }$ & $\sqrt{ }$ & $\times$ \\
\hline 5 & $\times$ & $\sqrt{ }$ \\
\hline 6 & $\sqrt{ }$ & $\times$ & $\sqrt{ }$ \\
\hline 7 & $\sqrt{ }$ & $\sqrt{ }$ & $\sqrt{ }$ \\
\hline
\end{tabular}

Table 9. Optimization results under different objectives for 33-bus system

\begin{tabular}{c|c|c|c|c}
\hline \hline Case & $C^{\text {Loss }}$ & $C^{\mathrm{WTG}}$ & $C^{\mathrm{TR}}$ & Iter. \\
\hline 1 & 11.30 & 399.48 & 84.04 & 1 \\
2 & 12.82 & 0 & 56.02 & 11 \\
3 & 13.26 & 0 & 46.58 & 1 \\
4 & 12.82 & 0 & 56.02 & 1 \\
5 & 13.26 & 0 & 46.58 & 1 \\
6 & 13.26 & 0 & 46.58 & 1 \\
7 & 13.26 & 0 & 46.58 & 1 \\
\hline \hline
\end{tabular}

Table 9 gives the optimization results of different cases using the base case data in the 33-bus system where the amount of renewable energies is less than that of the load demand. As is revealed, wind power curtailment only appears in Case 1 where power loss is the only optimization objective. High penetration of wind power may lead to larger power loss in Case 1. Case 2 and Case 4 share the same cost while the model in Case 2 converges in 11 iterations because the objective in Case 2 is just the minimization of wind power curtailment which is not monotonously increasing in power injections or branch current. In fact, the solution for Case 3 is also another optimal solution for Case 2, which demonstrates that the model in Case 2 has multiple optimal solutions. Moreover, all the results for Case 3, Case 5, Case 6 and Case 7 are 
the same. In some sense, the minimization the curtailment of wind power is equivalent to minimization of electricity purchasing from main grid.

Then the performance of the proposed cutting planes is verified based on the 69-bus system, where the total forecast wind power is $4.0 \mathrm{MW}$ which is larger than the load demand. The results for the 69-bus system are shown in Table 10.

Table 10. Optimization results under different objectives for 69-bus system

\begin{tabular}{c|c|c|c|c}
\hline \hline Case & $C^{\text {Loss }}(\$)$ & $C^{\mathrm{WTG}}(\$)$ & $C^{\mathrm{TR}}(\$)$ & Iter. \\
\hline 1 & 12.01 & 760.97 & 46.72 & 2 \\
2 & 14.57 & 112.38 & 23.74 & 15 \\
3 & 13.69 & 514.57 & 23.74 & 9 \\
4 & 14.61 & 112.38 & 23.74 & 10 \\
5 & 14.61 & 112.38 & 23.74 & 5 \\
6 & 12.20 & 518.39 & 23.74 & 17 \\
7 & 14.61 & 112.38 & 23.74 & 12 \\
\hline \hline
\end{tabular}

We can observe from Table 9 and Table 10 that the proposed cutting planes are effective in Case 2, Case 3 and Case 5, where the power loss is not included in the optimization objective. From the results of Table 10, although the power loss is the unique objective in Case 1, the relaxation is still not exact in the first trial, as shown in Fig.4. However, the model can frequently converge after the cut (15) integration.

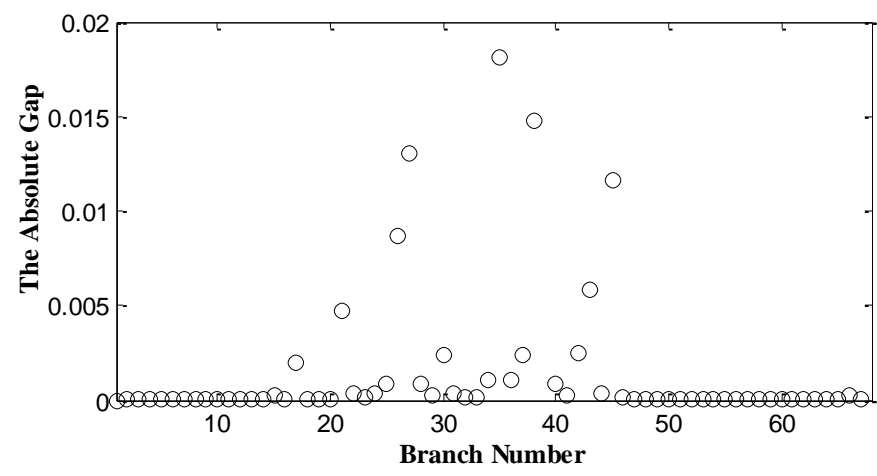

Fig. 4. Gap scatter without cutting planes in the first iteration.

Because of the main-grid constraints, the cost for electricity purchasing from the main-grid is $\$ 23.74$ for Cases 2-7, among which there are some differences in power 
losses and wind power curtailments. So, minimization of electricity purchasing from the main grid is not equivalent to minimization of wind power curtailment when the main-grid constraints are imposed.

Table 11. The relative maximum gap of SOCR in each iteration

\begin{tabular}{c|c|c|c}
\hline \hline Iteration & $\Delta_{k}^{\text {diff }}$ & Iteration & $\Delta_{k}^{\text {diff }}$ \\
\hline 1 & 11766.7799 & 7 & 0.0652 \\
2 & 3.2853 & 8 & 0.0613 \\
3 & 0.4306 & 9 & 0.0415 \\
4 & 0.2459 & 10 & 0.0255 \\
5 & 0.1227 & 11 & 0.0144 \\
6 & 0.0593 & 12 & 0.0098 \\
\hline \hline
\end{tabular}

To further explore the performance of cutting planes, the detailed information in each iteration is depicted in Fig. 5 and Table 11. It is clearly shown in Fig. 5 that the total cost can drastically increase after the first cutting planes integration, which can be seen in the results of the second iteration. We can also discover that although these objectives converge to satisfactory results around the 8-th step, the relative gap of SOCR in the corresponding step is not exact to computation criterion, as shown in Table 11.

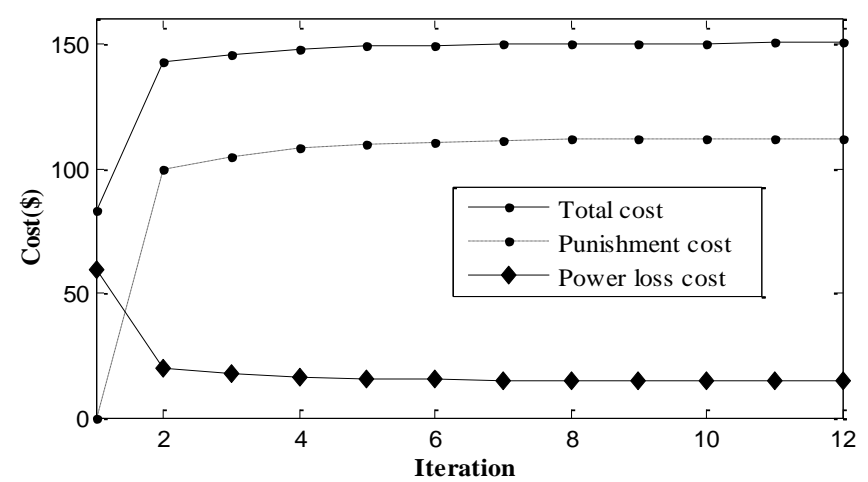

Fig. 5. Cost in each iteration for Case 7 in 69-bus system.

Besides, the cutting planes in [28] where cut (15) is excluded is applied to verify the improvement of proposed cuts. When applying the cutting planes in [28], the maximum relative gap of SOCR in the 50-th iteration of model in Case 7 is still 0.1393 shown in Fig. 6. As depicted, the inexact branches are mainly those leaf ones in the network. On 
the contrary, after cut (15) is integrated into the relaxed model the relaxation gaps finally meet the convergence criterion with less number of iterations (12 iterations in Case 7) presented in Table 10.

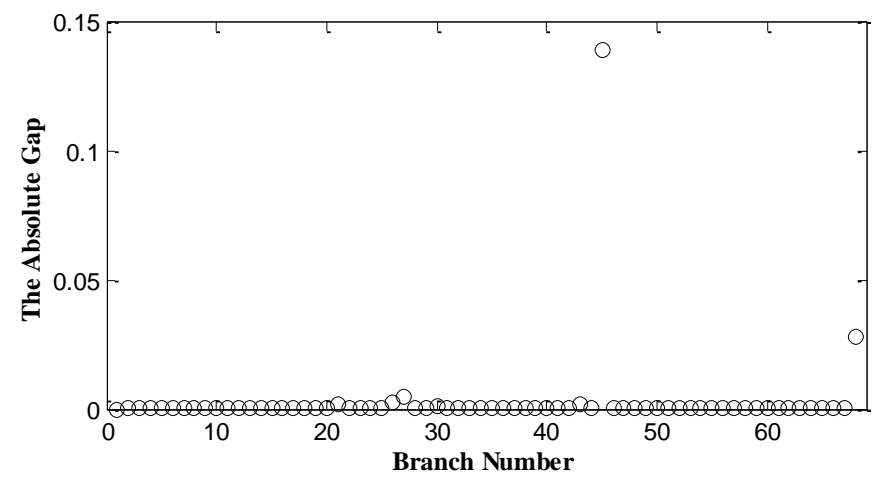

Fig. 6. Gap scatter without cut (15) in the 50-th iteration.

From the previous analysis, it can be concluded that the cutting planes including (15) and (18) lead to an improved performance in guaranteeing the relaxation exactness in almost every situation. In addition, the performances for the bus-69 system in Case 7 based on different algorithms are shown in Table 12.

Table 12. Comparison of solution performance by different algorithms for the 69-bus system

\begin{tabular}{c|c|c|c|c}
\hline \hline Problem-solving method & Type & $C(\$)$ & Time & Iteration \\
\hline This study & SOCP & 150.73 & $2.52 \mathrm{~s}$ & 12 \\
[28] & SOCP & $>152.59$ & $>5.82 \mathrm{~s}$ & $>50$ \\
IPOPT & NLP & 149.30 & $15.14 \mathrm{~s}$ & 3562 \\
GA & NLP & - & $>0.5 \mathrm{~h}$ & - \\
\hline \hline
\end{tabular}

From Table 12, we can clearly observe that the method proposed in this study is able to guarantee the model convergence within a finite number of iterations. But the model cannot converge in 50 iterations for the cutting planes in [28]. Compared the solution method proposed in this study and [28], Interior Point Optimizer (IPOPT) demands more computing time although it can obtain better optimization result (149.30). This implies that after the cutting planes are introduced, the global optimal solution may not 
be ensured even though the relaxed model is essentially related to a convex programming problem. However, the proposed method is still promising and applicable because it converges to the optimal solution in much less time as compared with IPOPT and genetic algorithm (GA), and its optimization result (150.73) is also acceptable. So the proposed method offers a desirable tradeoff between solution quality and computing cost. For Case 7 of 33-bus system (a simpler network), the optimal result can be obtained without any cutting planes, which can be found in Table 9. The comparative results of the obtained solutions for Case 7 of 33-bus system using the proposed method and IPOPT are presented in Table 13, which demonstrate that SOCP is of high-efficiency and is able to ensure that the global optimal solution is achieved if the SOC relaxation is exact.

Table 13. Comparison of the derived solutions between this study and IPOPT for the 33-bus system

\begin{tabular}{c|c|c|c|c}
\hline \hline Problem-solving method & Type & $C(\$)$ & Time & Iteration \\
\hline This study & SOCP & 59.84 & $0.51 \mathrm{~s}$ & 1 \\
IPOPT & NLP & 59.84 & $2.73 \mathrm{~s}$ & 84 \\
\hline \hline
\end{tabular}

\section{Conclusion}

This work introduce two types of cutting planes to ensure the exactness of SOCR in the OPF of active distribution system. The case studies are performed to demonstrate the effectiveness of the proposed method by a typical operation model. The conclusions are summarized as follows.

1) The cutting planes are suitable for the OPF with multiple PV nodes. They are necessary for relaxation exactness especially when the amount of renewable energies exceeds the load demand. And the model needs much fewer iterations when the reverse 
power flow is allowable because of larger feasible regions. This facilitates the practical application of BFM-ROPF in the next-generation ADS.

2) The proposed cutting planes are effective for Case 2, Case 3 and Case 5, where the power loss is not included in the optimization objective. They are applicable for general conditions in the radial distribution system.

3) The cutting planes combining (15) and (18) lead to an improved performance in guaranteeing the relaxation exactness in almost every situation, while (18) itself cannot sufficiently ensure the relaxation exactness in finite iterations.

In addition, the OPF proposed in this paper is mainly applicable to the optimal operation in the single time slot. The extension of BFM-ROPF to the practical problems based on multiple time slots (e.g. day-ahead dispatch) will be further studied in the future research.

\section{Acknowledgments}

This work was supported in part by the National High Technology Research and Development Program of China under Grant 2014AA051901, and in part supported by the National Science Foundation of China under Grant 51377111.

\section{References}

[1] J. Liu, H. Gao, Z. Ma, et al., Review and prospect of active distribution system planning, Journal of Modern Power Systems and Clean Energy. 3(Dec.) (2015) $457-467$. 
[2] Peng, Qiuyu, and Steven H. Low, Distributed Optimal Power Flow Algorithm for Balanced Radial Distribution Networks, 2015. [Online]. Available: http://arxiv.org/abs/1404.0700.

[3] Ferreira, Rafael S., Carmen LT Borges, and Mario VF Pereira, A flexible mixed-integer linear programming approach to the AC optimal power flow in distribution systems, IEEE Trans. Power Syst. 29 (2014) 2447-2459.

[4] T. Ding, S. Liu, W. Yuan, et al., A two-stage robust reactive power optimization considering uncertain wind power integration in active distribution networks, IEEE Trans Sustainable Enery. 7 (2016) 301-311.

[5] A. Keane, L. F. Ochoa, C. T. L. Borges, et al., State-of-the-art techniques and challenges ahead for distributed generation planning and optimization, IEEE Trans Power Syst. 28 (2013) 1493-1502.

[6] L. L. Lai, J. T. Ma, Improved genetic algorithms for optimal power flow under both normal and contingent operation states, Int J Electr Power Energy Syst. 19 (1997) $287-292$.

[7] M. A. Abido, Optimal power flow using tabu search algorithm, Electr Power Components Syst, 30 (2002) 469-483.

[8] M. Gomez-Gonzalez, A. Lopez, F. Jurado, Optimization of distributed generation systems using a new discrete PSO and OPF, Electr Power Syst Res, 84 (2012) $174-180$.

[9] A. Garces, A quadratic approximation for the optimal power flow in power distribution systems, Electr Power Syst Res, 130 (2016) 222-229. 
[10]D. Sun, B. Ashley, B. Brewer, et al., Optimal power flow by Newton approach, IEEE Transactions on Power Appar and Syst, 103 (1984) 2864-2880.

[11]M. Liu, S. K. Tso, Y. Cheng, An extended nonlinear primal-dual interior-point algorithm for reactive-power optimization of large-scale power systems with discrete control variables, IEEE Trans Power Syst, 17 (2002) 982-991.

[12]H. Wei, H. Sasaki, J. Kubokawa, et al., An interior point nonlinear programming for optimal power flow problems with a novel data structure, IEEE Trans Power Syst, 13 (1998) 870-877.

[13] Amini, M. Hadi, et al, Distributed security constrained economic dispatch, in: 2015 IEEE Innovative Smart Grid Technologies (ISGT), 2015.

[14]X. Ba, H. Wei, K. Fujisawa, et al., Semidefinite programming for optimal power flow problems, Int J Electr Power Energy Syst, 30 (2008) 383-392.

[15] Molzahn, Daniel K., and Ian A. Hiskens, Sparsity-exploiting moment-based relaxations of the optimal power flow problem, IEEE Transactions on Power Systems 30 (2015) 3168-3180.

[16] Molzahn, Daniel K., and Ian A. Hiskens, Mixed SDP/SOCP moment relaxations of the optimal power flow problem, in: 2015 IEEE Eindhoven PowerTech, 2015.

[17] S. H. Low, Convex relaxation of optimal power flow part I: formulations and equivalence, IEEE Trans Control of Network Syst, 1(2014) 15-27.

[18] J. Lavaei, S. H. Low, Zero duality gap in optimal power flow problem, IEEE Trans Power Syst, 27 (2012) 92-107. 
[19] M. Farivar, S. H. Low, Branch flow model: relaxations and convexification part I, IEEE Trans Power Syst, 28 (2013) 2554-2564.

[20] Jabr, Rabih A: Radial distribution load flow using conic programming, IEEE Trans Power Syst, 21 (2006) 1458-1459.

[21] M. Farivar, S. H. Low, Branch flow model: relaxations and convexification part II, IEEE Trans Power Syst, 28 (2013) 2565-2572.

[22] M. Farivar, C. R. Clarke, S. H. Low, et al., Inverter VAR control for distribution systems with renewables, 2011 IEEE International Conference on Smart Grid Communications, Brussels, Belgium, 2011, 17-20.

[23] Kocuk, Burak, Santanu S. Dey, and Xu Andy Sun. Inexactness of SDP relaxation and valid inequalities for optimal power flow, IEEE Trans Power Syst, 31 (2016) 642-651.

[24] Lavaei, Javad, David Tse, and Baosen Zhang. Geometry of power flows and optimization in distribution networks, IEEE Trans Power Syst, 29 (2014) 572-583.

[25]L. Gan, N. Li, U.Topcu, S. Low, On the exactness of convex relaxation for optimal power flow in tree networks, in: 2012 IEEE 51st IEEE Conference on Decision and Control (CDC), 2012, 465-471.

[26]N. Li, L. J. Chen, and S. H. Low, Exact convex relaxation of OPF for radial networks using branch flow model, in: IEEE Third Int Conference on Smart Grid Communications, 2012, 5-8.

[27]L. W. Gan, N. Li, U. Topcu, et al., Exact convex relaxation of optimal power flow in radial networks, IEEE Trans Automatic Control, 60 (2015) 72-87. 
[28] S. Y. Abdelouadoud, R. Girard, F. P. Neirac, et al., Optimal power flow of a distribution system based on increasingly tight cutting planes added to a second order cone relaxation, Int J Electr Power Energy Syst, 69 (2015) 9-17.

[29] Pindyck, Robert S, Uncertainty in the theory of renewable resource markets, The Review of Economic Studies 51 (1984) 289-303.

[30] Sarwat, Arif I., et al, Smart Grid reliability assessment utilizing Boolean Driven Markov Process and variable weather conditions, in: IEEE North American Power Symposium (NAPS), 2015.

[31] Gopalakrishnan, Ajit, et al. Global optimization of optimal power flow using a branch \& bound algorithm, In: IEEE 50th Annual Allerton Conference on Communication, Control, and Computing, Allerton, 2012.

[32]M. E. Baran and F. F. Wu, Optimal capacitor placement on radial distribution systems, IEEE Trans. Power Del., 4 (1989) 725-734.

[33]M. E. Baran and F. F. Wu, Optimal sizing of capacitors placed on a radial distribution system, IEEE Trans. Power Del., 4 (1989) 735-743.

[34] Y. Liu, W. Wu, B. Zhang, et al., A mixed integer second-order cone programming based active and reactive power coordinated multi-period optimization for active distribution network, Proceedings of the CSEE, 34 (2014) 2575-2583. 